\title{
Anti-Diabetics Drug Usage and Related Pharmaceutical Care Issues among Geriatrics and Non-Geriatrics: A Comparative Assessment
}

\author{
Syed Zia Inamdar*, Raghavendra Vinayakarao Kulkarni \\ Department of Pharmacy Practice, BLDEA's SSM College of Pharmacy and Research Centre, Vijaypur-586 103, Karnataka, INDIA.
}

\begin{abstract}
Background and Introduction: Diabetes Mellitus presents a challenge and continues to be a focus of medical care as population continues to age and live longer. The treatment approach in elderly and younger patients is influenced by ageing, life expectancy; co morbidities and severity of the vascular complications which increases the risk for development of pharmaceutical care issues. The present study evaluates anti-diabetic medication use and related pharmaceutical care issues among geriatric and non-geriatric population. Methods: A longitudinal cohort study was conducted in patients admitted to tertiary care hospital in north Karnataka with type 2 diabetes mellitus of age 18 years and above. Patient's demographic, clinical characteristics and anti-diabetic medications were reviewed to detect and report any pharmaceutical care issue using Pharmaceutical Care Network Europe checklist. Beers criteria were also utilized for geriatrics assessment. Results: A total of 230 diabetic [geriatric $53.48 \%$ and non-geriatric $46.52 \%$ ] patients were finally assessed for the study. Of the total drugs prescribed, anti-diabetic drugs category shared around $25.74 \%$ [geriatrics $15.26 \%$ and $10.48 \%$ in non-geriatrics]. A total of 147 pharmaceutical care issue related to anti diabetics were reported $[69.38 \%$ in geriatrics and $30.61 \%$ in non-geriatrics], which are related to; drug not appropriate for therapy [geriatrics $35.29 \%$ non-geriatrics 22.22 ], Contraindications related [geriatrics $26.47 \%$ non geriatrics $17.77 \%$ ], adverse drug reactions [geriatrics $6.86 \%$ non-geriatrics $6.66 \%$ ] and drug interaction [geriatrics $23.53 \%$ non-geriatrics $20 \%$ ]. Discussion: The geriatric population's social factors, thoughts and behavioral patterns, old age and concomitant diseases obscure diabetes management and contribute for the increased frequency of pharmaceutical care issues compare to non-geriatrics. Conclusion: The study shows a higher incidence of pharmaceutical care issues in geriatrics compare to nongeriatrics. The treatment plan for diabetes in geriatrics is not dissimilar to that of non-geriatrics. An individualized glycemic goals and interdisciplinary care is the key for optimal management of diabetes in different age groups.
\end{abstract}

Key words: Geriatric, Non-geriatric, Anti diabetic agents, Pharmaceutical care issues.

\section{INTRODUCTION}

The World health organization [WHO] describes Diabetes Mellitus (DM) as a metabolic ailment of multiple causes, distinguished by persistent increase in blood sugar with changes in carbohydrate, fat and protein metabolism which takes place due to disorders with synthesis of insulin, insulin activity, or both. A geographical disparity with unexplained cause was noted distinctly as most of the diabetics in Indian subcontinent are from age group 45-64 years whereas in developed countries the highly diabetic prevalent ages are more than 65 years of age. ${ }^{1}$

\section{DOI: 10.5530/ijopp.10.3.35}

$\mathrm{DM}$ is a complex, chronic illness which requires continuous medical care with multi-factorial risk reduction strategies beyond glycemic control. ${ }^{2}$ The elderly population is rapidly increasing in the world. In India at the year 2000 there were almost $4 \%$ of adults suffering from diabetes and it is expected that the incidence rate will increase to $6 \%$ in $2025 .{ }^{3}$ Although pharmacotherapies gratify $85 \%$ of patient's population, lifestyle modification presents itself as a potential source of treatment. ${ }^{4}$ In addition, most DM patients are having other health disorders along with it among which the most common are increased blood sugar
Address for correspondence: Dr. Syed Zia Inamdar, Department of Pharmacy Practice, BLDEA's SSM College of Pharmacy and Research, Centre Vijaypur-586 103, Karnataka INDIA.

Phone no: +91-9986666016 E-mail: syedzia.inamdar@gmail. com

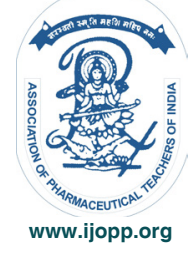


and hyperlipidemia. Due to this mostly patients should take numerous medications of about five to nine for maintaining better health and for better outcomes.

The treatment targets are same for younger and old age patients; although treatment plan are taken based on the increase in blood sugar and other risk factors like age, average life time expected, concomitant conditions and rigorousness of the vascular complications. ${ }^{5,6}$ The development of diabetic care plan and its management is an exceptional challenge in the geriatric patients with a prerequisite of initial comprehensive assessment and due consideration to pharmacokinetic, pharmacodynamic, and economic aspects. ${ }^{7}$

The main reason of death among diabetes patients is due to cardiovascular disorders i.e. almost $60-80 \%$. The prospective study of diabetes conducted in unitedkingdom (UKPDS) says that if the blood sugar levels are controlled as near to normal it can reduce the risk of cardiovascular disorders and other complications in diabetic patients. ${ }^{8}$ It has been postulated that metformin might promote Coronary artery disease $[\mathrm{CAD}]^{9}$ and is additionally having the risk of developing lactic acidosis, particularly in patients who have encountered with heart attack recently. The patients with heart related disorders should avoid taking sulphonylureas or glinides and should prefer other treatment options. Renal diseases increase the chances of developing reduced blood sugar levels in diabetes patients. It is because there is reduction in clearance by kidney during renal disease which leads to build up of antidiabetic drugs since there is reduced need of insulin. ${ }^{9,10}$ So the recommended drugs to use in case of renal disease with diabetes are glitazones, insulin, glinides or Dipeptidyl peptidase-4 (DPP-4) inhibitors with adjustments in dose. ${ }^{11}$

When a patient is having diabetes with liver disorders like non-alcoholic fatty liver disease (NAFLD) the chances of developing hypoglycemia is high, and the treatment will become difficult because most of the oral antidiabetic drugs are contraindicated and therefore doses must be adjusted in such patients. The diabetes type 2 patients with hepatic disorders are having very high chances of developing cirrhosis of liver, hepatic failure or cancer in liver. ${ }^{12}$

Diabetic patients have higher chances of developing stroke and when compared to non-diabetic population they have double risk for developing stroke. Studies shows that almost $20 \%$ of diabetic patients may die from stroke, which can make it as the main cause of death in diabetic patients. ${ }^{13}$ The principal reason for many diabetic patients not achieving the normal blood glucose target is hypoglycemia. If the patient has developed severe hypoglycemia in the past then sufonylureas, glinides and insulin should not be given, and less rigorous blood sugar targets should be kept. ${ }^{14,15,16}$ Starting insulin therapy is a very important stage in the management of type $2 \mathrm{DM}$. Most of the diabetic patients are not able to achieve the target Glycated hemoglobin [A1C] levels by single therapy using oral antidiabetic medications. Type $2 \mathrm{DM}$ has a progressive nature which leads to slow destruction to the $\beta$-cell function and its cell mass which eventually leads the patient to the initiation of insulin for the treatment. ${ }^{17,18}$ Most of the oral antidiabetic drugs are safe and effective when given in combination with insulin. When metformin is given in combination with insulin there are reduction in side effects like weight gain and hypoglycemia compared with insulin monotherapy. Thiazolidinedione's helps in decreasing insulin resistance but leads to side effects like increased weight, retention of fluid and chances of developing heart failure when given in combination with insulin. ${ }^{19}$ The present study evaluates anti diabetics medication usage and related pharmaceutical care issues among geriatric and non-geriatric study population.

\section{METHODS}

A longitudinal cohort study was conducted in a tertiary care hospital in north karnataka. Type $2 \mathrm{DM}$ patients of age 18 years and above $[\geq 60$ as geriatric and $\leq 60$ years as non-geriatrics] with or without concurrent illness were included in the study. Institutional ethics committee has approved the study design. The demographic details of the patient, clinical details, drug therapy, anti diabetic medication prescription were reviewed and assessed for the presence any pharmaceutical care issue. Pharmaceutical Care Network Europe [PCNE] checklist was utilized to assess pharmaceutical care issue with the use of anti-diabetic medication in the both patient population. A Beers criterion was also adopted in geriatrics assessment in addition to PCNE. The data collected was finally computed and reported in percentage using Microsoft word excel sheet 2007.

\section{RESULTS}

A total of 230 diabetic patients were included for the study of which geriatric were $123[76(61.78 \%)$ male and 47(38.21\%) female] and non-geriatric 107 [58(59.79\%) male and $39(40.21 \%)$ female]. Out of the total geriatric subjects enrolled majority 56.09\% were from 60-69 years age and in non-geriatrics $83.05 \%$ were of $41-60$ years of age. The major co morbidities observed in male and female geriatrics was Cardio vascular system[CVS] disorders [male $56.55 \%$ and female $48.6 \%$ ], followed by 
Respiratory disorders, diabetic complications, Central nervous system[CNS], Renal disorders, Gastrointestinal and others etc. Table 1. The major co morbidities observed in male and female non-geriatrics was CVS disorders [male 50\% and female 41.3\%], followed by diabetic complications, Respiratory disorders, Renal disorders, Gastrointestinal and others etc. Table 1.

Of all the drugs prescribed in the study population, around $25.74 \%$ share was of antidiabetic drugs, of which $15.26 \%$ [Oral hypoglycemic agents [OHA] 10.98\% and $4.28 \%$ insulin] in geriatrics with [8.41\% as monotherapy and $2.57 \%$ as combination] and $10.48 \%$ [OHA $5.20 \%$ and $5.28 \%$ insulin] in non-geriatrics [3.42\%as monotherapy and $1.78 \%$ as combination]. Majority of insulin as Monotherapy was prescribed in both geriatrics and nongeriatrics patient population Table 2 .

A total of 147[102 potential and 45 actual] Pharmaceutical care issues[PCI] related to antidiabetics were reported [126 in geriatrics and 21 in non-geriatrics] in the study population. Of which are related to drug without indication [geriatrics 3.92\% non-geriatrics 2.22\%] indication without drug [geriatrics $1.96 \%$, non-geriatrics
$6.66 \%$ ] drug not appropriate for therapy [geriatrics $35.29 \%$ non-geriatrics 22.22 ], contraindications [geriatrics $26.47 \%$ non geriatrics $17.77 \%$ ] supratherapeutics dose [geriatrics $0.98 \%$ non-geriatrics $2.22 \%$ ] additive toxicity [geriatrics $2.94 \%$ non-geriatrics $2.22 \%$ ] adverse drug reactions [geriatrics $6.86 \%$ non-geriatrics $6.66 \%$ ], drug interaction [geriatrics $23.53 \%$ non-geriatrics $20 \%$ ] and drug duplication [geriatrics $2.94 \%$ non- geriatrics $8.88 \%$ ] Table 3.

\section{DISCUSSION}

The study shows that the percentage of geriatric male patients $(61.78 \%)$ and non-geriatric male patients $(59.79 \%)$ diagnosed with type 2 diabetes mellitus was more compared to their female counterparts $(38.21 \%)$ and (40.21\%). Type 2 diabetes mellitus rate was observed to be more in the patients of age 60-70 years in both male $(56.09 \%)$ and female $(65.30 \%)$ geriatric patients, whereas in non-geriatric patients type 2 diabetes was more within the age group of $41-60$ years in both male $(81.03 \%)$ and female $(87.17 \%)$ patients Table 1.

\begin{tabular}{|c|c|c|c|c|c|}
\hline \multicolumn{6}{|c|}{ Patient Characteristics } \\
\hline & \multicolumn{2}{|c|}{ Geriatric $n=123$} & & \multicolumn{2}{|c|}{ Non geriatric $n=107$} \\
\hline \multirow[t]{2}{*}{ Gender } & Male[\%] & Female [\%] & & Male [\%] & Female [\%] \\
\hline & $76[61.78]$ & $47[38.21]$ & & $64[59.79]$ & $43[40.21]$ \\
\hline Mean sd \pm & $68.91 \pm 7.35$ & $68.72 \pm 7.39$ & & $49.73 \pm 8.11$ & $49 \pm 9.08$ \\
\hline Age group & \multicolumn{2}{|c|}{ Geriatrics } & & \multicolumn{2}{|c|}{ Non Geriatrics } \\
\hline distribution & $\begin{array}{c}\text { Male [\%] } \\
n=76\end{array}$ & 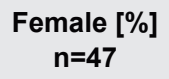 & & $\begin{array}{c}\text { Male [\%] } \\
n=64\end{array}$ & $\begin{array}{c}\text { Female [\%] } \\
n=43\end{array}$ \\
\hline $60-70$ & $41[53.95]$ & $29[61.70]$ & $18-20$ & $1[1.57]$ & -- \\
\hline $71-80$ & $20[26.32]$ & $16[34.04]$ & $21-40$ & $11[17.19]$ & $6[13.96]$ \\
\hline $81-90$ & $15[19.74]$ & $2[4.26]$ & $41-59$ & $52[81.25]$ & $37[86.05]$ \\
\hline Associated & \multicolumn{2}{|c|}{ Geriatrics } & & \multicolumn{2}{|c|}{ Non Geriatrics } \\
\hline Co-morbidities & $\begin{array}{c}\text { Male [\%] } \\
\qquad n=76\end{array}$ & $\begin{array}{c}\text { Female [\%] } \\
n=47\end{array}$ & & $\begin{array}{c}\text { Male [\%] } \\
\quad n=64\end{array}$ & $\begin{array}{c}\text { Female [\%] } \\
n=43\end{array}$ \\
\hline CVS & $69[56.55]$ & $36[48.6]$ & & $41[50]$ & $24[41.3]$ \\
\hline $\begin{array}{l}\text { Diabetes } \\
\text { complication }\end{array}$ & $14[11.47]$ & $15[20.2]$ & & $14[17.07]$ & $15[25.86]$ \\
\hline CNS & $07[5.73]$ & $11[14.86]$ & & $05[6.09]$ & --- \\
\hline $\begin{array}{l}\text { Respiratory } \\
\text { disorders }\end{array}$ & $18[14.75]$ & $05[6.7]$ & & $04[4.87]$ & $08[13.79]$ \\
\hline Renal disorders & $04[3.27]$ & $03[4.05]$ & & $03[3.65 \%]$ & $01[1.72]$ \\
\hline $\begin{array}{l}\text { Gastrointestinal } \\
\text { disorders }\end{array}$ & 02 [1.63] & 02[2.70] & & $06[7.31 \%]$ & $01[1.72]$ \\
\hline Anemia & $04[3.27]$ & $02[2.70]$ & & --- & ----- \\
\hline others & $04[3.27]$ & ---- & & $09[10.97]$ & $09[15.51]$ \\
\hline
\end{tabular}

CVS; Cardiovascular system disorders, CNS; Central nervous system disorders 
Table 2: Pattern of Oral Hypoglycemic Agents and Insulin used in Geriatrics and Non-geriatrics population.

OHA

Monotherapy

Metformin

Gliclazide

Voglibose

Glimepiride

Glipizide

Pioglitazone

Repaglinide

Combination

Glimipride + metformin

Glipizide + Metformin

Voglibose + Metformin

Pioglitazone + Metformin

Gliclazide + metformin

Glibenclamide + metformin

Nateglinide + metformin

Glimepride+pioglitazone+Metformin

Insulin

Monotherapy

Actrapid

Recosulin

Basalog

Lupisulin

Insugen

Huminsulin

Combination

Mixtard
Geriatric $n=214 \quad$ Non-geriatric $n=147$

$$
\mathrm{n}=118
$$

$\mathrm{n}=48$

$53[44.91 \%]$

$31[64.5 \%]$

$09[7.62 \%]$

$06[12.5 \%]$

$08[6.77 \%]$

$03[6.25 \%]$

06[5.08\%]

03[6.25\%]

$06[5.08 \%]$
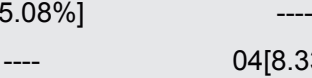

04[8.33\%]

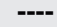

$\mathrm{n}=36$

01[2.08\%]

$\mathbf{n}=\mathbf{2 5}$

$15[41.66 \%]$

07[28\%]

$12[33.33 \%]$

03[12\%]

$03[8.33 \%]$

02[5.55\%]

01[4\%]

$01[2.77 \mathrm{~s} \%]$

01[4\%]

$---$

$09[36 \%]$

03[8.33\%]

01[4\%]

$$
\mathrm{n}=60
$$

$03[12 \%]$

$\mathrm{n}=74$

38 [63.33\%]

35[47.29\%]

09[15\%]

$21[28.37 \%]$

04[6.66\%]

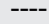

$04[6.66 \%]$

$01[1.35 \%]$

$01[1.66 \%]$

$06[8.10 \%]$

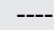

$04[5.40 \%]$

$04[6.66 \%]$

07[9.45\%]

Table 3: Pharmaceutical Care Issues identified in Geriatrics and Non-Geriatrics study population.

Pharmaceutical Care Issues $n=147$

Pharmaceutical care issues category

Drug without indication

Indication without drug

Drug not appropriate for therapy

Contraindications

Supratherapeutic dose

Additive toxicity

Suspected ADR's

Drug - Drug interaction

Drug Duplication

Total PCI
Geriatrics n=102

\section{Male}

3

1

21

18

1

3

2

14

2

65
Female

1

1

10

9

9

0

0

5

10

1

37

3.92

1.96

30.39

26.47

0.98

2.94

6.87

23.53

2.94

$100 \%$
Non-Geriatrics $n=45$

Female

$\%$

0

2.22

6.66

33.33

17.77

2.22

2.22

6.66

20

8.88

19

$100 \%$

$\mathrm{PCl}_{\text {; Pharmaceutical Care Issues }}$ 
The most common co-morbid condition associated with type $2 \mathrm{DM}$ in both gender geriatric patients and non-geriatric patients was found to be CVS disorders $[56.55 \%, 48.6 \%$ and $50 \%, 41.3 \%]$ Followed by diabetic induced complications, respiratory and, renal disorders etc. Table 1.

The study observed most commonly used OHA monotherapy agent was metformin (44.91\%), followed by sulfonylurea $(17.78 \%)$ and in combination therapy was Metformin with Glimepiride (41.66\%) in geriatrics, which was also observed in non-geriatrics with monotherapy of metformin $(64.5 \%)$, followed by sulfonylureas $(18.75 \%)$ and combination therapy was Metformin with Glimepiride (286\%). This indicates that metformin and glimepride+ metformin was the choice of drug in both geriatrics and adults. But the usage of metformin was more in adult population. Human Actrapid was found to be the most commonly used insulin for type 2 diabetes mellitus therapy among both geriatric $(63.3 \%)$ and nongeriatric $(47.29 \%)$ patients. Table 2.

The study observed a total of 147 different pharmaceutical care issues [PCD in both geriatrics [69.39\%] and nongeriatrics $[30.61 \%]$. Frequency of PCI was higher in geriatrics subjects compare to non-geriatrics; the probable reason could be, ageing characteristics of the subjects, co-morbidities and multiple medication use, which increases the risk of development of PCI. It has been postulated that metformin might promote $\mathrm{CAD}$ and can also have an increased chance of leading to development of lactic acidosis mostly in patients encountered with heart attack recently. ${ }^{9}$ In cardiac muscle cells and smooth muscle cells in arteries, Adenosine triphosphate - sensitive potassium channel $\left[K_{\text {ATP }}\right.$ channels] are seen abundantly. Sulfonylurea's increases the secretion of insulin by binding to the $\mathrm{K}_{\text {ATP }}$ channels in pancreatic $\beta$-cell; therefore, there are many chances that these drugs can bind to $\mathrm{K}_{\text {ATP }}$ channels in cardiac muscle cells and vascular smooth muscle cells. In cardiac muscle cells the $\mathrm{K}_{\text {ATP }}$ channels intercede for preconditioning of ischemia. The exposure of cardiac muscle cells to various episodes of ischemia can induce changes in cell adaptations thus making these cells resistant to injuries during consequent ischemic attacks 6 . There were 19 geriatrics and 7 nongeriatric cases were identified in which sulfonylureas were used in CAD. Table 3.

Sulfonylureas are generally not to be recommended in renal impairment. Because during kidney diseases there will be Prolonged half-life with reduced renal excretion of Sulfonyl urea which has been shown to increase the incidence of reduced blood sugar level complexities (hypoglycemia) to about five times more than in patients without kidney disease. ${ }^{9}$ In this study a case of geriatric patient in which sulfonylureas (glipizide) was used in renal impairment was observed Table 3.

The use and contraindications of antidiabetic drugs during cerebrovascular disease matches generally with cardiovascular disease recommendations. ${ }^{8}$ During cerebro vascular disease all the antidiabetic drugs except sulphonylureas and glinides can be used for the diabetic therapy. A few geriatric cases were identified where Sulfonylureas were used in stroke Table 3. Nicotinamide Adenine Dinucleotide $\left[\mathrm{NAD}^{+}\right]$required to convert lactate to pyruvate is supplied during gluconeogenesis. The antidiabetic drugs from biguanide class inhibit gluconeogenesis in the liver and kidney which increases the chance of development of lactic acidosis if the liver functions are impaired. Metformin induced hepatotoxicity cases are only a few but because of chances of development of lactic acidosis with metformin use in liver impaired patients, metformin is contraindicated in advanced hepatic disorder patients. A geriatric and a non-geriatric case were identified where metformin was used in liver impairment Table 3. Since Sulfonylureas are not expensive drugs and are well tolerated by patients, it is commonly used antidiabetic drug and it is among the first oral antidiabetic drugs developed. They usually act by stimulating the synthesis of insulin from pancreas and thus show its anti-diabetic effect. ${ }^{13}$ A few (4) geriatric cases were identified where sulfonylureas were used in hypoglycemia Table 3 . The study reveals around $15.21 \%$ of total OHA prescribed in both geriatrics and non-geriatrics were inappropriate in terms of drug selection, dosage regimen, patient preferences and against established practice of therapy owing to possible physician preference, evidence based or peer practice. The study reveals that there was a wide usage of insulin and sulfonylureas among the geriatrics which was generally considered to be unsafe, because both the treatments ultimately lead to severe hypoglycemia. 67 geriatric cases were identified for inappropriateness in therapy, among which 25 cases had inappropriateness in insulin prescription where insulin was prescribed with Fasting blood glucose $[\mathrm{FBG}] \leq 250 \mathrm{mg} / \mathrm{dl}$ and 42 cases had inappropriateness in prescription of sulfonylurea Table 3. It was reasonable approach to initiate treatment with oral agents in most of the patients, with an exemption for the patients with severe hyperglycemia (Plasma glucose in empty stomach $>250 \mathrm{mg} / \mathrm{dl}$ ). Such patients need insulin therapy, or basal bolus insulin treatment to reduce blood sugar levels. In other case starting oral treatment can be very much successful ${ }^{14}$ Table 3.

The study also observed episodes of adverse drug effect with use of antidiabetic, with majority in geriatric females 
(5 episodes), followed by geriatric males (2 episodes) and male non-geriatrics ( 3 episodes) with events ranging from hypoglycemia [insulin, glimepride] to metallic taste [metformin]. The study reveals potential drug interaction in the study population. The geriatrics was observed with higher number (24) of drug interaction compare to nongeriatrics (9). The interaction observed was of mild to moderate in nature, but the risk was higher in geriatrics patients due to poly pharmacy or increased number of drug use Table 3.

\section{CONCLUSION}

The study reveals similar pattern of disease management in older and younger diabetic patients using antidiabetic drugs with some exemption. Advance age, nutrition, behavioral and social factors, and other concomitant medical conditions promotes the complexity of diabetes management in elderly when compared to the younger patients influencing increased risk for the precipitation of drug related problems and pharmaceutical care issues. The key for optimal management of diabetes should involve a comprehensive assessment of diabetic patient to bring about individualized drug therapy in the context of ageing phenomenon, complexity of diseases and concurrent illness to ensure safe and effective treatment outcome.

\section{ACKNOWLEDGEMENT}

The authors are thankful to the management of BLDE association for supporting the work.

\section{CONFLICT OF INTEREST}

No conflict of interest.

\section{REFERENCES}

1. Acharya KG, Shah KN, Solanki ND, Rana DA. Evaluation of antidiabetic prescriptions, cost and adherence to treatment guidelines: A prospective, cross-sectional study at a tertiary care teaching hospital. Journal of Basic and Clinical Pharmacy. 2013;4(4):82-7.

2. Standards of Medical Care in Diabetes-2014. Position Statement. American Diabetes Association. Diabetes Care. 2014;37(1):S14-S80.

3. Day C. The rising tide of type 2 diabetes. British Journal of Diabetes and Vascular Disease. 2001;1(1):37-43.

4. Reduction in the Incidence of Type 2 Diabetes with Lifestyle Intervention or Metformin. Diabetes Prevention Programmed Research Group. The New England Journal of Medicine. 2002;346(6):393-403.

5. Vigersky RA. An Overview of Management Issues in Adult Patients with Type 2 Diabetes Mellitus. Journal of Diabetes Science and Technology. 2011;5(2):245-50.

6. McCulloch DK, Munshi M, Treatment of type 2 diabetes mellitus in the older patient. [Cited 2016]. Available at: http://www.uptodate.com/contents/ treatment-of-type-2-diabetes-mellitus-in-the-older-patient.

7. Mooradian AD, McLaughlin S, Boyer CC, Winter J. Diabetes Care for Older Adults. Diabetes Spectrum.1999;12(2):70-7.

8. Caulfield MT, O'Brien KD. Cardiovascular Safety of Oral Antidiabetic Agents: The Insulin Secretagogues. Clinical Diabetes. 2002;20(2):81-4.

9. Misbin RI, Green L, Stadel BV, Gueriguian JL, Gubbi A, Fleming GA. Lactic Acidosis in Patients with Diabetes Treated with Metformin. The New England Journal of Medicine. 1998;338(4):265-6.

10. Tschope D, Hanefeld M, Meier JJ, Gitt AK, Halle M, Bramlage P et al. The role of co-morbidity in the selection of antidiabetic pharmacotherapy in type-2 diabetes. Cardiovascular Diabetology. 2013;12(1):62.

11. Sherlan Thomas et al. Managing type 2 diabetes with renal impairment. Clinical advisor. 2011.

12. Gupte P, Amarapurkar D, Agal S, Baijal R, Kulshrestha P, Pramanik S, et al. Nonalcoholic steatohepatitis in type 2 diabetes mellitus. Journal of gastroenterology and hepatology. 2004;19(8):854-8.

13. Phypers B, Pierce JMT. Lactate physiology in health and disease. Continuing Education in Anaesthesia, Critical Care and Pain. 2006;6(3):128-32.

14. Hewitt J, Guerra LC, Moreno MCF, Sierra C. Diabetes and Stroke Prevention: A Review. Stroke Research and Treatment. 2012:6. doi:10.1155/2012/673187.

15. Campbell IW. Sulfonylureas and hypoglycemia. Diabetic Hypoglycemia. 2009;2(1):3-10.

16. Hirsch IB, Bergenstal RM, Parkin CG, Wright E, Buse JB. A Real-World Approach to Insulin Therapy in Primary Care Practice. Clinical Diabetes. 2005;23(2):78-86.

17. Henske JA, Griffith ML, Fowler MJ. Initiating and Titrating Insulin in Patients with Type 2 Diabetes. Clinical Diabetes. 2009;27(2):72-6.

18. McCulloch DK. Diabetes mellitus type 2: Treatment (beyond basics). [cited 2016].Available at: http://www.uptodate.com/contents/diabetes-mellitus-type2-treatment-beyond-the-basics.

19. Petznick A. Insulin Management of Type 2 Diabetes Mellitus. American Family Physician. 2011;84(2):183-90. 